\title{
Fermion condensation induced by the Weyl anomaly
}

\author{
Chong-Sun Chu $\odot^{2,3}$ and Rong-Xin Miao $\oplus^{1, *}$ \\ ${ }^{1}$ School of Physics and Astronomy, Sun Yat-Sen University, Zhuhai 519082, China \\ ${ }^{2}$ National Center for Theoretical Sciences, National Tsing-Hua University, Hsinchu 30013, Taiwan \\ ${ }^{3}$ Department of Physics, National Tsing-Hua University, Hsinchu 30013, Taiwan
}

(Received 26 April 2020; accepted 24 July 2020; published 14 August 2020)

\begin{abstract}
Fermi condensation is usually a phenomena of strongly correlated system. In this paper, we point out a novel mechanism for condensation of Dirac fermions due to the Weyl anomaly. The condensation has its physical origin in the nontrivial response of the fermion vacuum to changes in the background spacetime (either boundary location or the background metric), and can be felt when a background scalar field is turned on. The scalar field can be, for example, the Higgs field in a fundamental theory or the phonon in condensed matter system. For a spacetime with boundaries, the induced Fermi condensate is inversely proportional to the proper distance from the boundary. For a conformally flat spacetime without boundaries, Fermi condensation depends on the conformal factor and its derivatives. We also generalize the Banks-Casher relation which relates the Fermi condensate to the zero mode density of the Dirac operator to a local form. Due to its universal nature, this anomaly induced Fermi condensate can be expected to have a wide range of applications in physics.
\end{abstract}

DOI: $10.1103 /$ PhysRevD.102.046011

\section{INTRODUCTION}

Fermi condensation $\langle\bar{\psi} \psi\rangle \neq 0$ is an interesting quantum phenomena and has a wide range of applications. The Cooper pair in BCS theory of superconductivity is a famous example of Fermi condensation. It is the bound state of a pair of electrons in a metal with opposite spins. The chiral condensate of massless fermions is another example of Fermi condensation. In QCD the chiral condensate is an order parameter of transitions between different phases of quark matter in massless limit. The condensation of fermionic atoms has been observed in experiment [1]. The condensation of fermion is usually attributed to the effects of strongly coupled dynamics and hence it can be used as an order parameter characterizing the phases of the theory. A motivation of this work is to investigate if there is any novel and universal mechanism other than strongly coupled dynamics that can give rises to Fermi condensation.

Recently a novel phenomena of induced current was predicted in the quantum field theory of Dirac fermions coupled to external electromagnetic field

\footnotetext{
*Corresponding author. miaorx@mail.sysu.edu.cn

Published by the American Physical Society under the terms of the Creative Commons Attribution 4.0 International license. Further distribution of this work must maintain attribution to the author(s) and the published article's title, journal citation, and DOI. Funded by SCOAP.
}

$$
S=\int_{M} \sqrt{-g}\left(\bar{\psi} i \gamma^{\mu} \nabla_{\mu} \psi+\bar{\psi} \gamma^{\mu} A_{\mu} \psi\right)
$$

It was found that an applied magnetic field will give rise to a nonuniform magnetization density of the vacuum and induces a magnetization current

$$
\left\langle J^{\mu}\right\rangle=\frac{-2 \beta F^{\mu \nu} n_{\nu}}{x}+\cdots, \quad x \sim 0
$$

in the vicinity of the boundary of the vacuum system $[2,3]$. Here $\beta$ is the beta function, $x$ is the proper distance to the boundary, $n_{\mu}$ is the inner normal vector and ... denote higher order terms in $O(x)$. Note that the universal results (2) works for general quantum field theory and not just conformal field theory. In a conformally flat spacetime $d s^{2}=e^{2 \sigma} \eta_{\mu \nu} d x^{\mu} d x^{\nu}$ without boundaries, the anomalous current is given by $[4,5]$

$$
\left\langle J^{\mu}\right\rangle=-2 \beta F^{\mu \nu} \partial_{\nu} \sigma+O\left(\sigma^{2}\right),
$$

to the leading order of small $\sigma$. Generalization of the result (2) to higher dimensions and the result (3) for arbitrary finite $\sigma$ can be found in [6-8] respectively. We remark that these anomalous currents do not rely on the presence of a material system, but is a pure vacuum phenomena. This is completely different from the other well-known transport phenomena [9-16] that is due to chiral anomaly and a finite chemical potential is needed. In fact, the anomalous current (2) can be regarded as a kind of magnetic Casimir effect 
since it arises from the nontrivial electromagnetic response of the vacuum to the change of boundary (location). This is very similar to the Casimir effect which originated from the mechanical response of the vacuum to the change of the boundary (location). As for (3), it arises from the fact that the vacuum of the theory is different for different $\sigma$ and a nonvanishing vev for the current operator is resulted due to nontrivial Bogoliubov transformation. This is similar to the process of particle creation during cosmological expansion or the Hawking radiation [17].

Motivated by these results for the anomalous current, it is natural to consider other couplings of the fermion to external field, and one can expect similar induced phenomena to occur. In this paper, we show that, in addition to currents, Weyl anomaly can give rises to Fermi condensation if a background scalar field is turned on. The resulting Fermi condensate is a nontrivial function of space. In the standard situation of a Dirac operator coupled to external vector field in a flat spacetime, the Fermi condensate is translational invariant and it is well known to be related to the density of zero modes of the Dirac operator by the Bank-Casher relation [18]. For more general Dirac operator, we show that the Fermi condensate obeys an elegant generalized form of the Bank-Casher relation, see (24) and (25) below.

\section{WEYL ANOMALY AND FERMI CONDENSATE}

Let us start with the action of Dirac fermion $\psi$ coupled to a scalar field $\phi$ in a curved spacetime with metric $g_{\mu \nu}$ :

$$
S=\int_{M} \sqrt{-g}\left(i \bar{\psi} \gamma^{\mu} \nabla_{\mu} \psi+\phi \bar{\psi} \psi\right)
$$

In this paper, we use the signature $(1,-1,-1,-1)$ for the metric and the scalar field will be taken as a background and so whether there is a Lagrangian for the scalar field is irrelevant to us. In this theory, the renormalized expectation value of the Fermi condensate $\langle\bar{\psi} \psi\rangle$ can be derived by the variation of effective action with respect to the background scalar field

$$
\langle\bar{\psi} \psi\rangle=\frac{1}{\sqrt{-g}} \frac{\delta I_{\mathrm{eff}}}{\delta \phi} .
$$

The action (4) is classically Weyl invariant under the local scaling transformation: $\psi \rightarrow e^{-\sigma} \psi, g_{\mu \nu} \rightarrow e^{2 \sigma} g_{\mu \nu}$ and $\phi \rightarrow e^{-\sigma} \phi$. However, quantum mechanically there is an anomaly. Imposing the bag boundary conditions $[19,20]$ $\left.\left(1 \pm \gamma_{5} \gamma_{n}\right) \Psi\right|_{\partial M}=0$ and applying the heat kernel expansion [21], we obtain the Weyl anomaly $\mathcal{A}\left(g_{\mu \nu}, \phi\right)$ at one loop as

$$
\begin{aligned}
\mathcal{A}= & \frac{1}{8 \pi^{2}}\left(\int_{M} \sqrt{-g}\left[-(\nabla \phi)^{2}+\frac{R \phi^{2}}{6}+\phi^{4}\right]\right. \\
& \left.+\int_{\partial M} \sqrt{-h} \frac{k \phi^{2}}{3}\right) .
\end{aligned}
$$

Here $R$ is Ricci scalar in the bulk $M, h_{i j}$ is the induced metric on the boundary $\partial M, k_{i j}$ is the extrinsic curvature and $k$ is its trace. Note that we have ignored the gravitational contribution to Weyl anomaly, i.e., $\int_{M} O\left(R^{2}\right)+$ $\int_{\partial M} O(R k)$, since they are independent of $\phi$ and hence are irrelevant for the Fermi condensation. In the following, we show that the knowledge of the Weyl anomaly (6) allows one to determine the Fermi condensate in closed analytic form without performing any perturbative QFT calculation. This is one of the main results of this paper.

\section{FERMI CONDENSATION I: BOUNDARY THEORY}

Let us first study the Fermi condensation in 4-dimensional spacetime with a boundary, say, at $x=0$ of a certain coordinate system. We follow the methods of [2,22], where we have studied the expectation value of current and stress tensor in boundary quantum field theories [23]. As the mass dimension of $\psi$ is $3 / 2$, the Fermi condensate takes the asymptotic form [24]

$$
\langle\bar{\psi} \psi\rangle=\frac{O_{0}}{x^{3}}+\frac{O_{1}}{x^{2}}+\frac{O_{2}}{x}+O(\ln x)
$$

near the boundary. Here $x$ is the proper distance from the boundary. $O_{\mathrm{n}}$ have mass dimension $\mathrm{n}$ and depend on only the background geometry and the background scalar field. For example, we have $O_{0}=c_{0}, O_{1}=c_{1} \phi+c_{2} k$, where $c_{i}$ are numbers. Since Weyl anomaly is related to the UV Logarithmic divergent term of effective action, one can follow the same analysis performed in [2,22] and obtain the following "integrability" relation [25]

$$
(\delta \mathcal{A})_{\partial M}=\left(\int_{M} \sqrt{-g}\langle\bar{\psi} \psi\rangle \delta \phi\right)_{\log \epsilon}
$$

between the renormalized Fermi condensate and the boundary part of the variation of the Weyl anomaly. Here a regulator $x \geq \epsilon$ to the boundary has been introduced for the integral on the right-hand side (rhs) of (8). For our purpose, we turn only on the variation of the scalar field. Using (8), one can derive Fermi condensate near the boundary from the boundary terms of variations of the Weyl anomaly. To proceed, let us employ the Gauss normal coordinates to write the metric $d s^{2}=d x^{2}+\left(h_{i j}-2 x k_{i j}+\cdots\right) d y^{i} d y^{j}$ and expand $\phi(x)=\phi_{0}+x \phi_{1}+x^{2} \phi_{2}+O\left(x^{3}\right)$, where $x \in$ $[0,+\infty)$ and $\phi_{i}$ give the $i$ th derivatives of $\phi$ at $x=0$. 
From (6), we get for the left-hand side (lhs) of (8)

$$
(\delta \mathcal{A})_{\partial M}=\frac{-1}{4 \pi^{2}} \int_{\partial M} \sqrt{-h}\left(\nabla_{n} \phi-\frac{1}{3} k \phi\right) \delta \phi_{0},
$$

where $n$ denotes the internal normal of the manifold $M$. Next, we substitute (7) into the rhs of (8), integrate over $x$ and select the logarithmic divergent term, we obtain

$$
\int_{\partial M} \sqrt{-h}\left[-O_{0} \delta \phi_{2}+\left(k O_{0}-O_{1}\right) \delta \phi_{1}-\left(O_{2}+\cdots\right) \delta \phi_{0}\right],
$$

where $\cdots$ denotes terms which depend on $O_{0}$ and $O_{1}$ which vanish when $O_{0}=O_{1}=0$. Comparing (9) and (10), we obtain $O_{0}=O_{1}=0$ and the Fermi condensation near the boundary:

$$
\langle\bar{\psi} \psi\rangle=\frac{1}{4 \pi^{2}} \frac{\nabla_{n} \phi-\frac{1}{3} k \phi}{x}+O(\ln x), \quad x \sim 0 .
$$

Several comments are in order.

(1) Similar to the case of current and stress tensor [2,22], the Fermi condensate is finite at the boundary since there are boundary contributions to the Fermi condensation which cancel the divergence from the bulk contribution (11).

(2) The result (11) is for the bag boundary conditions at one loop. For general boundary conditions, there are corrections to Weyl anomaly (6) and $O_{0}$ and $O_{1}$ of (7) are nonzero [26].

(3) The result (11) for the bag boundary condition, as well as the general form (7) for general boundary conditions can be reproduced in holographic theory [26].

(4) Similar to [2,22], the above discussions on Weylanomaly-induced Fermi condensation apply not only to conformal field theory (CFT) but also the general quantum field theory (QFT). That is because Weyl anomaly is well defined for general quantum field theories [27,28].

(5) If $\phi$ is the Higgs field and get a vev $\phi=-m$, then the fermion get a mass $m$ and the chiral symmetry is spontaneous broken. Our analysis implies that a Fermi condensate is induced near a curved boundary

$$
\langle\bar{\psi} \psi\rangle=\frac{1}{12 \pi^{2}} \frac{m k}{x}, \quad x \sim 0
$$

due to the Higgs phenomena.

(6) Note that the above discussions apply to more general theories instead of just the free theory (4). In fact, there are universal relations between the Fermi condensate and Weyl anomaly [26].

Similar to the origin for the induced current discussed in $[2,3]$, the existence of the Fermi condensation (11) can be understood in terms of the changed properties of the vacuum due to the presence of boundary. For simplicity let us consider the case of a half space flat geometry $x \geq 0$. From the form of the action (4), the fermions see a potential of $-\phi$ and experience a force

$$
F_{x}=\partial_{x} \phi
$$

in the $x$-direction. This may also be derived from the Ehrenfest theorem $\left\langle\frac{d \mathcal{O}}{d t}\right\rangle=\frac{1}{i \hbar}\langle[\mathcal{O}, H]\rangle$ for the time evolution of the observable $\mathcal{O}$. For the Hamiltonian $H=$ $-\int d^{3} x \bar{\psi}\left(i \gamma^{i} \partial_{i}+\phi\right) \psi$, we obtain the "Newton's law" $\left\langle\frac{d p_{x}}{d t}\right\rangle=\partial_{x} \phi\langle\bar{\psi} \psi\rangle$ for the $x$-direction, and $\left\langle d p_{a} / d t\right\rangle=0$ for $a=y, z$. This gives the force (13) on single particle states. For simplicity, let us consider the case of a constant force $\partial_{x} \phi>0$. Consider the pair creation process at any point $P$ in space. Due to the presence of an upward force (for the discussion here, we will call the positive $x$ direction as upward), the particles which are created to move upward will, for the same period of time $\Delta t$, travel a longer distance compared to the particles which are created to move downward. Note that the force does not differentiate particles and antiparticles and so the number density $\rho_{P}$ for the fermions and antifermions contributed by source point $P$ will be affected by the force the same way. As a result, $\rho_{P}$ will not be symmetric with respect to $P$, but is skewed toward the negative $x$ direction. See Fig. 1. Now take any observation point $O$ in space. The amount of condensate at $O$ is obtaining by summing over the contribution from all source points $P$. When there is no boundary, obviously a constant condensate $\langle\bar{\psi} \gamma\rangle\rangle$ is created. Things are different when there is a boundary at $x=0$. For a source point $P$ situated near the boundary, the particles that are created to move downward will have less chance to be annihilated since the space $x<0$ are all absent now and hence there are less particles that can travel up to annihilate

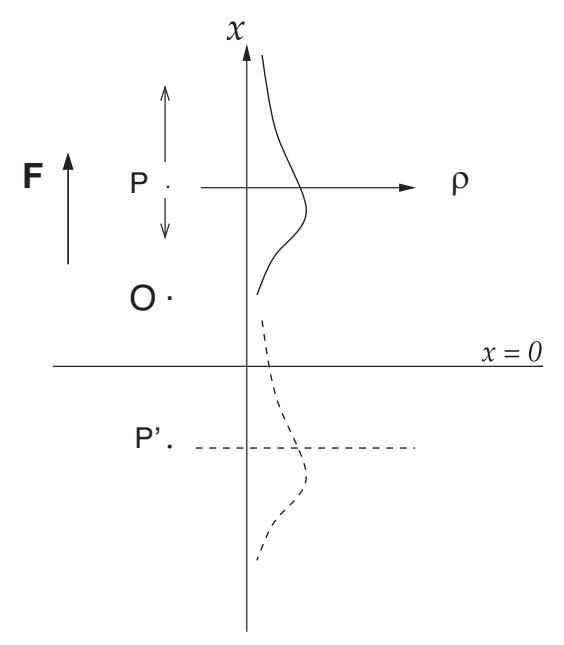

FIG. 1. Fermi condensate near the boundary. The contribution from $P^{\prime}$ is absent when half space $x>0$ is considered. 
them. As a result, the distribution of particles will be skewed even more toward the boundary. Also the skewing is greater as one gets closer to the boundary. Summing up the contributions from all source points, one see immediately that the condensate is bigger toward the boundary. Also it is positive and is proportional to the force $\partial_{x} \phi$. Obviously the same discussion holds for the case of $\partial_{x} \phi<0$. Qualitatively, we find exact agreement with the result (11). It is worth noting that since the scalar force is the same for the fermions and the antifermions, the virtual pairs are pushed toward the boundary in the same manner and there is no separation of charges. Charge neutrality of the vacuum is maintained.

It is interesting to remark that the condensate (11) of fermions near the boundary can also be understood as a kind of Bose-Einstein condensation with localization in coordinate space. Heuristically, due to the force (13), the virtual particles experience an acceleration $a=F_{x} / m$ and hence an Unruh temperature [29]

$$
T=|a| /(2 \pi k) .
$$

For the discussion here, we will allow for both signs of $\partial_{x} \phi$. Since the condensate is a bosonic state and obeys the BoseEinstein statistics, the mean occupation number of the condensate is given by $N=1 /\left(e^{E / k T}-1\right)$. Now the particles created at a location $x$ has a potential energy of $E \sim F_{x} x$. Near the boundary we have $E \ll k T$ and $N \sim k T / E \sim \operatorname{sgn}\left(\partial_{x} \phi\right) / m x$. The condensate $\langle\bar{\psi} \psi\rangle$ has mass dimension 3 and should depend on $m$ and $\partial_{x} \phi$. The natural relation $\langle\bar{\psi} \psi\rangle \sim m\left|\partial_{x} \phi\right| \times N$ then gives precisely the result (11).

\section{FERMI CONDENSATION II: CONFORMALLY FLAT SPACETIME}

Fermi condensation can also occur in conformally flat spacetime without boundaries. To demonstrate this, let us start by deriving the anomalous transformation rule for the Fermi condensate. Consider the theory (4) with metric and scalar field given by $\left(g_{\mu \nu}, \phi\right)$. Due to the anomaly, the renormalized effective action $I_{\text {eff }}$ is not invariant under the Weyl transformation. Generally, we have [30] $\frac{\delta}{\delta \sigma} I_{\text {eff }}\left(e^{-2 \sigma} g_{\mu \nu}, e^{\sigma} \phi\right)=\mathcal{A}\left(e^{-2 \sigma} g_{\mu \nu}, e^{\sigma} \phi\right)$ for arbitrary finite $\sigma(x)$. This can be integrated to give the effective action [31-33]. Using the fact that the anomaly (6) is Weyl invariant up to a surface term: $\mathcal{A}\left(e^{-2 \sigma} g_{\mu \nu}, e^{\sigma} \phi\right)=$ $\mathcal{A}\left(g_{\mu \nu}, \phi\right)+\int_{M} \partial_{\mu}\left(\sqrt{-g} \phi^{2} g^{\mu \nu} \partial_{\nu} \sigma\right)$, we obtain immediately the transformation rule for the effective action:

$$
\begin{aligned}
& I_{\mathrm{eff}}\left(e^{-2 \sigma} g_{\mu \nu}, e^{\sigma} \phi\right) \\
& =I_{\mathrm{eff}}\left(g_{\mu \nu}, \phi\right) \\
& \quad+\frac{1}{8 \pi^{2}} \int_{M} \sqrt{-g}\left[\left(-(\nabla \phi)^{2}+\frac{R \phi^{2}}{6}+\phi^{4}\right) \sigma+\frac{\phi^{2}}{2}(\nabla \sigma)^{2}\right],
\end{aligned}
$$

plus a boundary term $\frac{1}{24 \pi^{2}} \int_{\partial M} \sqrt{-h} k \phi^{2} \sigma$, which we drop in spacetime without boundaries. One can check that the dilaton effective action satisfies Wess-Zumino consistency $\left[\delta_{\sigma_{1}}, \delta_{\sigma_{2}}\right] I_{\text {eff }}=0$. Using (15), we obtain finally the transformation rule for the Fermi condensate (5) under Weyl transformation $g_{\mu \nu} \rightarrow g_{\mu \nu}^{\prime}=e^{-2 \sigma} g_{\mu \nu}, \phi \rightarrow \phi^{\prime}=e^{\sigma} \phi$,

$$
\langle\bar{\psi} \psi\rangle=-\frac{1}{4 \pi^{2}}\left[\nabla(\sigma \nabla \phi)+\left(2 \phi^{3}+\frac{1}{6} \phi R\right) \sigma+\frac{1}{2} \phi(\nabla \sigma)^{2}\right],
$$

plus a trivial term $e^{-3 \sigma}\langle\bar{\psi} \psi\rangle^{\prime}$. Here $\langle\bar{\psi} \psi\rangle$ (resp. $\langle\bar{\psi} \psi\rangle^{\prime}$ ) denotes the vev of the Fermi condensate of the theory (4) in the background spacetime $g_{\mu \nu}$ (resp. $g_{\mu \nu}^{\prime}$ ). Taking $g_{\mu \nu}^{\prime}$ to be the flat spacetime metric and the fact that the Fermi condensation vanishes in flat spacetime, we finally obtain (16) as the Fermi condensate in conformally flat spacetime

$$
d s^{2}=e^{2 \sigma} \eta_{\mu \nu} d x^{\mu} d x^{\nu} .
$$

Several comments are in order. (1) The conformal factor $\sigma$ of (16) is arbitrary and needs not to be small. As a result, we can use (16) to calculate Fermi condensation in general conformally flat spacetimes such as antide Sitter space, de Sitter space and Robertson-Walker universe. (2) For Robertson-Walker universe $d s^{2}=$ $d t^{2}-a(t)^{2}\left(d x^{2}+d y^{2}+d z^{2}\right)$, we have at time $t=t_{*}$

$$
\langle\bar{\psi} \psi\rangle=-\frac{1}{4 \pi^{2}}\left(H \dot{\phi}+\frac{1}{2} H^{2} \phi\right)
$$

where dot denotes time derivative and $H=\dot{a} / a$ is the Hubble parameter. For simplicity we have chosen $a\left(t_{*}\right)=1$. (3) Unlike the case with boundaries, (16) works well only for CFT. At high energy scale such as early universe, the particle mass can be ignored and fermions can be regarded as CFT approximately. Then all of the above discussions apply. (4) The result (16) can also be derived for strongly coupled conformal field theory that is dual to gravity [26]. (5) The physical reason for the condensate (16) is simple. It arises because the vacuum of the theory in the spacetime (17) at a conformal factor $\sigma$ is no longer a vacuum as the metric changes to have a different conformal factor $\sigma^{\prime}$. As a result, a fermion condensate is created in a way similar to the creation of particles due to cosmological expansion (see for example, [17]).

\section{GENERALIZED BANKS-CASHER RELATION}

The Banks-Casher relation links the spontaneous breaking of chiral symmetry in QCD to the presence of a nonzero density of zero modes of the Dirac operator of the quark field. In ordinary consideration where the QFT is translational invariant, the condensate is a constant and one has the following relation [18] 


$$
\langle\bar{\psi} \psi\rangle=\pi \rho(0),
$$

where $\rho(\lambda)$ is the spectral density of the Dirac operator, see (22) below. Here let us generalize the Banks-Casher relation to the case where the condensate is not a constant. Consider a theory of Dirac fermions in curved space coupled to an external field $X$ :

$$
S=\int_{M} \sqrt{-g} i \bar{\psi} D \psi, \quad \text { where } i D:=i \gamma^{\mu} \nabla_{\mu}+X .
$$

For example, $X=\gamma^{\mu} A_{\mu}$ gives the minimal coupling to gauge potential $A_{\mu}, X=\phi$ gives the Yukawa coupling to a scalar field, and $X=i \gamma^{\mu \nu} F_{\mu \nu}$ gives the Pauli coupling to gauge field strength. Let $\psi_{n}$ be the eigenstate of the Dirac operator $i D$ with eigenvalue $\lambda_{n}$, and satisfies the orthogonormal relation $\int_{M} \sqrt{-g} \psi_{n}^{\dagger} \psi_{m}=\delta_{n m}$. It follows from the path integral definition of chiral condensate $\langle\bar{\psi} \psi\rangle=$ $Z^{-1} \int \mathcal{D} \psi \mathcal{D} \bar{\psi} e^{-S} \bar{\psi} \psi$ that

$$
\langle\bar{\psi} \psi\rangle=-\sum_{n} \psi_{n}^{\dagger}(x) \frac{1}{\lambda_{n}} \psi_{n}(x) .
$$

It is convenient to introduce the spectral density

$$
\rho(\lambda):=\frac{1}{V} \sum_{n} \delta\left(\lambda-\lambda_{n}\right),
$$

where $V$ is the spacetime volume. The relation (21) takes the form

$$
\frac{i}{V}\langle\bar{\psi} \psi\rangle=-\int_{-\infty}^{\infty} d \lambda \rho(\lambda) \frac{1}{\lambda+i \epsilon} \psi_{\lambda}^{\dagger}(x) \psi_{\lambda}(x),
$$

where a Wick rotation $\bar{\psi} \rightarrow i \bar{\psi}$ on the spinor has been performed to obtain the relation (23) in Minkowski space. We have introduced an $i \epsilon$ prescription $(\epsilon>0)$ to regulate the divergence due to the existence of zero modes of the operator $i D$. Using the relation $\frac{1}{\lambda+i \epsilon}=\mathrm{P} \frac{1}{\lambda}-i \pi \delta(\lambda)$, where $\mathrm{P}$ denote the Cauchy principal value, we obtain the following generalized Banks-Cashier relation in the unintegrated form:

$$
\frac{1}{V}\langle\bar{\psi} \psi(x)\rangle=\pi \rho(0)\left|\psi_{0}(x)\right|^{2}+i \mathrm{P} \int_{-\infty}^{\infty} d \lambda \rho(\lambda) \frac{1}{\lambda}\left|\psi_{\lambda}(x)\right|^{2} .
$$

This can also be integrated to give

$$
\frac{1}{V} \int_{M} \sqrt{-g}\langle\bar{\psi} \psi\rangle=\pi \rho(0)+i \mathrm{P} \int_{-\infty}^{\infty} d \lambda \frac{\rho(\lambda)}{\lambda} .
$$

For flat space, the condensate is a constant due to translational invariance. For operator which anticommute with $\gamma^{5}:\left\{i D, \gamma^{5}\right\}=0$ (e.g., minimal coupling to external gauge field), the eigenvalues of $i D$ always come in pairs $\pm \lambda$ and it is $\psi_{-\lambda}=\gamma^{5} \psi_{\lambda}$ for $\lambda \neq 0$. In this case, the continuum contribution in the rhs of (25) vanishes and we obtain the standard Banks-Cashier (19). For the general case of a curved spacetime with a non-minimal coupling, the condensate can become imaginary in general, and the condensate is related to the spectral density as in (24) and (25). For the case of Yukawa coupling in conformally flat spacetime, the condensate is real and we obtain the constraint

$$
\mathrm{P} \int_{-\infty}^{\infty} d \lambda \rho(\lambda) \frac{1}{\lambda}\left|\psi_{\lambda}(x)\right|^{2}=0
$$

even through the spectrum is not symmetric.

\section{CONCLUSIONS}

In this paper we have shown that the nontrivial vacuum structure of the theory of Dirac fermions in the presence of an external scalar field can lead to the occurrence of Fermi condensation. This is a direct consequence of the violation of scaling symmetry in the presence of a background scalar field that couples to the fermion. In general, it is also interesting to consider the effect of external pseudoscalar field on the Fermi condensation [26]. Due to the simple and universal nature of the Yukawa coupling, one can expect the Fermi condensation (11), (16) to find a wide range of physical applications. For example the possible occurrence of a condensate during the inflationary phase of the universe may have nontrivial consequences on various dynamical aspects of the early Universe such as the reheating, the evolution of physical structure, the pattern of symmetry realization etc. Experimentally, it may be possible to make observation of the Fermi condensate in optomechanical system with boundary or in a thermal system where temperature can be stimulated by a conformally flat background [34].

\section{ACKNOWLEDGMENTS}

We thank T. W. Chiu, B. L. Hu, S. Iso, S. Lin, and G. Shiu for useful discussions. C. S. C. was supported by NCTS and the Grant MOST 107-2119-M-007-014-MY3 of MSTT. R.X.M. acknowledges the NSFC Grant (No. 11905297). Note that all Institutes contribute equally to this work, the order of Institutes is adjusted for the assessment policy of SYSU. 
[1] C. A. Regal, M. Greiner, and D. S. Jin, Phys. Rev. Lett. 92, 040403 (2004).

[2] C. S. Chu and R. X. Miao, Phys. Rev. Lett. 121, 251602 (2018).

[3] C. S. Chu and R. X. Miao, J. High Energy Phys. 07 (2018) 005.

[4] M. N. Chernodub, Phys. Rev. Lett. 117, 141601 (2016).

[5] M. N. Chernodub, A. Cortijo, and M. A. H. Vozmediano, Phys. Rev. Lett. 120, 206601 (2018).

[6] C. Chu and R. Miao, J. High Energy Phys. 07 (2019) 151.

[7] C. Chu, Fortsch. Phys. 67, 1910005 (2019).

[8] J. J. Zheng, D. Li, Y. Q. Zeng, and R. X. Miao, Phys. Lett. B 797, 134844 (2019).

[9] A. Vilenkin, Astrophys. J. 451, 700 (1995).

[10] A. Vilenkin, Phys. Rev. D 22, 3080 (1980).

[11] M. Giovannini and M. E. Shaposhnikov, Phys. Rev. D 57, 2186 (1998).

[12] A. Y. Alekseev, V. V. Cheianov, and J. Frohlich, Phys. Rev. Lett. 81, 3503 (1998).

[13] K. Fukushima, Lect. Notes Phys. 871, 241 (2013).

[14] D. Kharzeev and A. Zhitnitsky, Nucl. Phys. A797, 67 (2007).

[15] D. T. Son and P. Surowka, Phys. Rev. Lett. 103, 191601 (2009).

[16] K. Landsteiner, E. Megias, and F. Pena-Benitez, Phys. Rev. Lett. 107, 021601 (2011).

[17] N. D. Birrell and P.C. W. Davies, Quantum Fields in Curved Space, Cambridge Monographs on Mathematical
Physics (Cambridge University Press, Cambridge, England, 1984).

[18] T. Banks and A. Casher, Nucl. Phys. B169, 103 (1980).

[19] A. Chodos, R. L. Jaffe, K. Johnson, C. B. Thorn, and V. F. Weisskopf, Phys. Rev. D 9, 3471 (1974).

[20] A. Chodos, R. L. Jaffe, K. Johnson, and C. B. Thorn, Phys. Rev. D 10, 2599 (1974).

[21] D. V. Vassilevich, Phys. Rep. 388, 279 (2003).

[22] R. X. Miao and C. S. Chu, J. High Energy Phys. 03 (2018) 046.

[23] J. L. Cardy, arXiv:hep-th/0411189.

[24] D. Deutsch and P. Candelas, Phys. Rev. D 20, 3063 (1979).

[25] Note that $\log \epsilon$ appears on the right-hand side of the relation (8) since we adopt here the signature convention $(1,-1,-1,-1)$. In contrast, the signature convention $(-1,+1,+1,+1)$ was adopted in [2] and $\log 1 / \epsilon$ appears.

[26] C. S. Chu and R. X. Miao, arXiv:2005.12975.

[27] M. J. Duff, Classical Quantum Gravity 11, 1387 (1994).

[28] L. S. Brown, Phys. Rev. D 15, 1469 (1977).

[29] W. Unruh, Phys. Rev. D 14, 870 (1976).

[30] We have find it conveniently to consider the Weyl transformation $g_{\mu \nu} \rightarrow e^{-2 \sigma} g_{\mu \nu}$ here.

[31] J. Wess and B. Zumino, Phys. Lett. 37B, 95 (1971).

[32] A. Cappelli and A. Coste, Nucl. Phys. B314, 707 (1989).

[33] A. Schwimmer and S. Theisen, Nucl. Phys. B847, 590 (2011).

[34] J. M. Luttinger, Phys. Rev. 135, A1505 (1964). 